Also available at http://amc.imfm.si

ISSN 1855-3966 (printed edn.), ISSN 1855-3974 (electronic edn.)

ARS MATHEMATICA CONTEMPORANEA 2 (2009) 101-119

\title{
Minimum cycle bases of direct products of graphs with cycles
}

\author{
Zachary Bradshaw * \\ Department of Mathematics and Applied Mathematics, Virginia Commonwealth \\ University, Richmond, VA, USA \\ Richard H. Hammack ${ }^{\dagger}$ \\ Department of Mathematics and Applied Mathematics, Virginia Commonwealth \\ University, Richmond, VA, USA
}

Received 17 July 2008, accepted 18 May 2009, published online 22 June 2009

\begin{abstract}
We construct a minimum cycle basis for the direct product $G \times C_{q}$ where $G$ is a connected non-bipartite graph, $C_{q}$ is an odd cycle and $G \times C_{q}$ is triangle-free. These bases are expressed in terms of the cycle structure of the symmetric digraph on $G$.
\end{abstract}

Keywords: Graph direct product, minimum cycle bases, digraphs.

Math. Subj. Class.: O5C38

\section{Introduction}

In the article Minimum Cycle Bases of Product Graphs [7], W. Imrich and P. Stadler construct minimum cycle bases for Cartesian and strong products of graphs, in terms of minimum cycle bases of the factors. F. Berger [1] and M. Jaradat [8] solve the same problem for the lexicographical product. The corresponding construction for the direct product appears to be extremely complex. The problem has been solved for the special cases where both factors are bipartite [4] or complete graphs [2, 5]. The authors of the present paper have an outline of a construction for a minimum cycle basis of the product $G \times H$ where both factors are arbitrary connected non-bipartite graphs (and at least one factor is triangle-free), but the proofs are too long to be of much interest. In this paper we offer a scaled-back version of the problem. We describe minimum cycle bases for $G \times C_{q}$ where $G$ is a connected non-bipartite graph and $C_{q}$ is the odd cycle on $q$ vertices.

\footnotetext{
* Supported by the Virginia Commonwealth University Honors Summer Undergraduate Research Program.

${ }^{\dagger}$ Corresponding author.

E-mail addresses: bradshawz@vcu.edu (Zachary Bradshaw), rhammack@vcu.edu (Richard H. Hammack)
} 
We begin with a brief review of the preliminaries. The edge space $\mathcal{E}(G)$ of a simple graph $G=(V(G), E(G))$ is the power set of its edges $E(G)$ endowed with the structure of a vector space over the two-element field $\mathbb{F}_{2}=\{0,1\}$. Addition in $\mathcal{E}(G)$ is symmetric difference of sets, and zero is the empty set. To simplify notation, we blur the distinction between subgraphs $K$ of $G$ and their edge set $E(K) \in \mathcal{E}(G)$. Thus, if $J$ and $K$ are subgraphs, an expression such as $J+K$ means $E(J)+E(K)$, with the operation taking place in $\mathcal{E}(G)$. With this convention, we regard $E(G)$ as the set of all connected one-edge subgraphs of $G$, so $E(G)$ a basis for $\mathcal{E}(G)$. Similarly, the vertex space $\mathcal{V}(G)$ of $G$ is the power set of $V(G)$ endowed with a vector space structure over $\mathbb{F}_{2}$. (Addition is symmetric difference.) Bending the notation slightly (as was done for the edge space) we regard $V(G)$ as a basis for $\mathcal{V}(G)$.

The cycle space of $G$, denoted $\mathcal{C}(G)$, is the subspace of $\mathcal{E}(G)$ consisting of the edge sets $E(K)$ of Eulerian subgraphs $K$ of $G$, that is, subgraphs having no vertex of odd degree. (See [3], Proposition 1.9.2). Elements of $\mathcal{C}(G)$ are called cycles. We call a cycle a simple cycle if it is connected and each vertex has degree 2 . The simple cycle with $p$ edges is denoted $C_{p}$. The dimension of $\mathcal{C}(G)$ is the (first) Betti number $\beta(G)=|E(G)|-|V(G)|+c$, where $c$ is the number of components of $G$ ([3], Theorem 1.9.6). A graph homomorphism $g: G \rightarrow H$ induces a linear map $g^{*}: \mathcal{E}(G) \rightarrow \mathcal{E}(H)$ defined on the basis $E(G)$ as $g^{*}(v w)=g(v) g(w)$. It is easy to check that $g^{*}$ restricts to a linear map $g^{*}: \mathcal{C}(G) \rightarrow \mathcal{C}(H)$.

A basis $\mathcal{B}$ of $\mathcal{C}(G)$ is called a cycle basis of $\mathbf{G}$, and its length is $\ell(\mathcal{B})=\sum_{C \in \mathcal{B}}|C|$. Among all cycle bases of $G$, one with smallest possible length is called a minimum cycle basis, or MCB. It is easy to check that every MCB contains only simple cycles.

The cycle space is a weighted matroid where each element $C$ has weight $|C|$. Hence the Greedy Algorithm [10] always terminates with an MCB. (I.e. begin with $\mathcal{M}=\emptyset$; then append shortest cycles to it, maintaining independence of $\mathcal{M}$, until no further shortest cycles can be appended; then append next-shortest cycles, maintaining independence, until no further such cycles can be appended; and so on, until $\mathcal{M}$ is a maximal independent set. Then $\mathcal{M}$ is an MCB.)

Here is our primary criterion for determining if a cycle basis is an MCB.

Proposition 1.1. A cycle basis $\mathcal{B}=\left\{B_{1}, B_{2}, \ldots, B_{\beta(G)}\right\}$ for a graph $G$ is an $M C B$ if and only if every $C \in \mathcal{C}(G)$ is a sum of basis elements whose lengths do not exceed $|C|$.

Proof. Suppose $\mathcal{B}$ is an $\mathrm{MCB}$, but there is a cycle $C=\sum_{k=1}^{\beta(G)} b_{k} B_{k}$ (each $b_{k}$ is in $\mathbb{F}_{2}$ ) and $|C|<\left|B_{k}\right|$ for some $k$ with $b_{k} \neq 0$. Then we can exchange basis element $B_{k}$ for $C$ and obtain a basis with smaller total length than $\mathcal{B}$, contradicting minimality. Conversely, suppose $\mathcal{B}$ is not an MCB. Assume that the elements $B_{1}, B_{2}, \ldots$ are arranged in order of increasing length. Since the greedy algorithm cannot terminate with basis $\mathcal{B}$, there must be an element $B_{p}$ for which the set $\left\{B_{1}, B_{2}, \ldots, B_{p-1}\right\}$ can be extended to an independent set $\left\{B_{1}, B_{2}, \ldots, B_{p-1}, C\right\}$ with $|C|<\left|B_{p}\right|$. Necessarily then, $C=\sum_{k=1}^{\beta(G)} b_{k} B_{k}$ with $b_{i} \neq 0$, for some $p \leq i \leq \beta(G)$, and $|C|<\left|B_{i}\right|$.

The direct product of graphs $G$ and $H$ is the graph $G \times H$ whose vertex set is the Cartesian product $V(G) \times V(H)$ and whose edges are $(u, x)(v, y)$ where $u v \in E(G)$ and $x y \in E(H)$. We quickly mention a few standard facts; the reader requiring more background is referred to [6]. Suppose $G$ and $H$ are connected. Then $G \times H$ is connected if and only if one of $G$ and $H$ has an odd cycle. Otherwise, if $G$ and $H$ are both bipartite then $G \times H$ has exactly two components. As $G \times H$ has $|V(G)| \cdot|V(H)|$ vertices and 
$2|E(G)| \cdot|E(H)|$ edges we have $\beta(G \times H)=2|E(G)| \cdot|E(H)|-|V(G)| \cdot|V(H)|+1$ whenever $G$ and $H$ are connected and one has an odd cycle.

Note that the standard projection maps $\pi_{G}: G \times H \rightarrow G$ and $\pi_{H}: G \times H \rightarrow H$ induce projection operators $\pi_{G}^{*}: \mathcal{C}(G \times H) \rightarrow \mathcal{C}(G)$ and $\pi_{H}^{*}: \mathcal{C}(G \times H) \rightarrow \mathcal{C}(H)$.

For any simple cycle $C_{p}$, we put $V\left(C_{p}\right)=\mathbb{Z}_{p}=\{0,1,2, \ldots, p-1\}$ and we agree that the edges of $C_{p}$ join $i$ to $i+1$ for each $i \in \mathbb{Z}_{p}$. Thus an arbitrary edge is written $i(i+1)$, which we take care not to confuse with multiplication.

\section{An MCB for $G \times K_{2}$}

To motivate our approach for constructing MCB's of $G \times C_{q}$, we now examine the problem of constructing an MCB for $G \times K_{2}$, where $G$ is an arbitrary connected graph and $K_{2}$ is the complete graph on 2 vertices. We put $V\left(K_{2}\right)=\{0,1\}$ and denote its edge as 01 .

We remark at the onset that an MCB of $G$ typically bears little resemblance to an MCB of $G \times K_{2}$. For example, consider Figure 1. The factor $G$ consists of a pentagon that shares a vertex with a triangle. Obviously, an MCB for $G$ consists of just two cycles, the pentagon and the triangle. But an MCB of $G \times K_{2}$ consists of two 8-gons (shown solid and dashed) plus the 6-gon over the triangle. The two 8-gons do not seem in any way related to any single element of the MCB for $G$. Our main task in this section is to uncover how an MCB for $G \times K_{2}$ is related to the structure of the factor $G$.

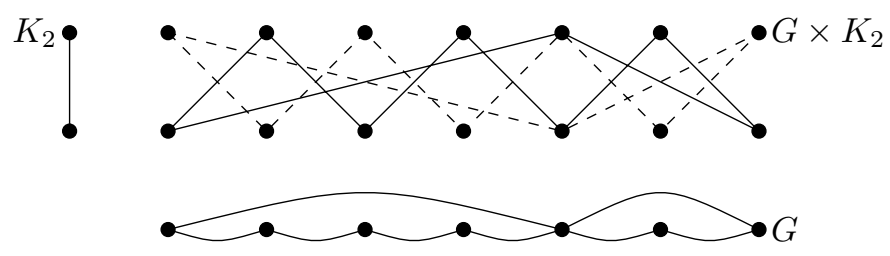

Figure 1: An example of $G \times K_{2}$

Our basic approach is to employ not the cycle structure of $G$, but instead the cycle structure of the symmetric digraph on $G$. Any graph $G$ can be identified with a symmetric digraph $\overleftrightarrow{G}$ obtained by replacing each edge $x y$ of $G$ with an arc $\overrightarrow{x y}$ directed from $x$ to $y$ and an arc $\overrightarrow{y x}$ directed from $y$ to $x$. Thus $\overleftrightarrow{G}$ has vertex set $V(\overleftrightarrow{G})=V(G)$ and arc set $E(\overleftrightarrow{G})=\{\overrightarrow{x y}, \overrightarrow{y x}: x y \in E(G)\}$. We define an anti-cycle to be a sub-digraph of $\overleftrightarrow{G}$, each vertex of which has even in-degree and even out-degree. Figure 2 shows an anti-cycle for the factor $G$ from Figure 1 . Let $\mathcal{A}(\overleftrightarrow{G})$ denote the set of anti-cycles in $\overleftrightarrow{G}$. In what follows we describe how $\mathcal{A}(\overleftrightarrow{G})$ is naturally identified with $\mathcal{C}\left(G \times K_{2}\right)$, and how "minimum anticycle bases" of $\mathcal{A}(\overleftrightarrow{G})$ correspond to MCB's of $G \times K_{2}$.

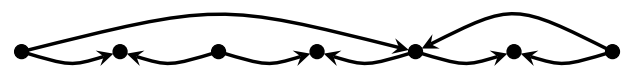

Figure 2: An anti-cycle in $G$

Let $\mathcal{E}(\overleftrightarrow{G})$ denote the power set of $E(\overleftrightarrow{G})$ endowed with a vector space structure over 
$\mathbb{F}_{2}$. (Addition is symmetric difference, etc.) As usual, to keep the notation under control we identify elements of $\mathcal{E}(\overleftrightarrow{G})$ with sub-digraphs of $\overleftrightarrow{G}$, so, for example, an element $\{\overrightarrow{x y}\} \in$ $\mathcal{E}(\overleftrightarrow{G})$ is written simply as $\overrightarrow{x y}$. With this convention, the set $E(\overleftrightarrow{G})$ is a basis for $\mathcal{E}(\overleftrightarrow{G})$ which has dimension $2|E(G)|$.

Define a linear map $\pi: \mathcal{E}\left(G \times K_{2}\right) \rightarrow \mathcal{E}(\overleftrightarrow{G})$ which acts on the basis $E\left(G \times K_{2}\right)$ as $\pi((x, 0)(y, 1))=\overrightarrow{x y}$. This is a vector space isomorphism because it sends the basis $E\left(G \times K_{2}\right)$ of $\mathcal{E}\left(G \times K_{2}\right)$ injectively onto the basis $E(\overleftrightarrow{G})$ of $\mathcal{E}(\overleftrightarrow{G})$. A moment's thought confirms that $\pi$ restricts to an isomorphism $\pi: \mathcal{C}\left(G \times K_{2}\right) \rightarrow \mathcal{A}(\overleftrightarrow{G})$.

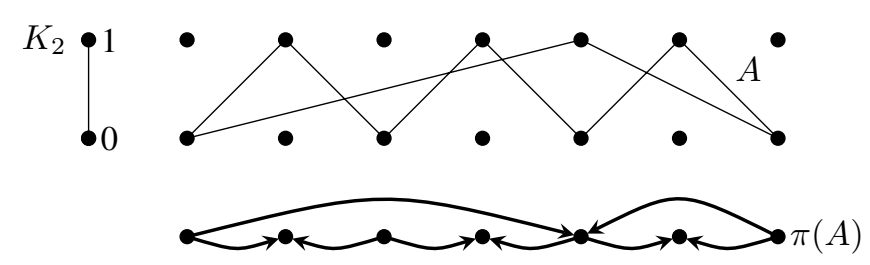

Figure 3: How anti-cycles in $G$ correspond to cycles in $G \times K_{2}$

Let the length $|C|$ of an element $C \in \mathcal{C}(\overleftrightarrow{G})$ be the number of arcs in $C$. Observe that $|A|=|\pi(A)|$ for every $A \in \mathcal{C}\left(G \times K_{2}\right)$. Thus $\pi$ is a length-preserving isomorphism from $\mathcal{C}\left(G \times K_{2}\right)$ to $\mathcal{A}(\overleftrightarrow{G})$

In analogy with the definition of an MCB, we define a minimum anti-cycle basis of $\overleftrightarrow{G}$ to be a basis $\left\{B_{1}, B_{2}, \ldots, B_{\beta\left(G \times K_{2}\right)}\right\}$ for $\mathcal{A}(\overleftrightarrow{G})$ in which the total length $\sum\left|B_{i}\right|$ has the smallest possible value. The remarks above show that $\pi$ gives an exact correspondence between minimum anti-cycle bases of $\overleftrightarrow{G}$ and minimum cycle bases of $G \times K_{2}$. This leads to the following result linking MCBs of $G \times K_{2}$ to the structure of $G$.

Proposition 2.1. Let $\pi: \mathcal{C}\left(G \times K_{2}\right) \rightarrow \mathcal{A}(\overleftrightarrow{G})$ be the isomorphism defined by the rule $\pi((x, 0)(y, 1))=\overrightarrow{x y}$. Then $\left\{B_{1}, B_{2}, B_{3}, \ldots, B_{\beta\left(G \times K_{2}\right)}\right\}$ is a minimum anti-cycle basis for $\overleftrightarrow{G}$ if and only if the set $\left\{\pi^{-1}\left(B_{1}\right), \pi^{-1}\left(B_{2}\right), \pi^{-1}\left(B_{3}\right), \ldots, \pi^{-1}\left(B_{\beta\left(G \times K_{2}\right)}\right)\right\}$ is an $M C B$ for $G \times K_{2}$.

We do not address the question of how to compute a minimum anti-cycle basis, since our main goal is to express an MCB of $G \times K_{2}$ in terms of invariants of the factors, and Proposition 2.1 accomplishes this in the sense that the structure of $\overleftrightarrow{G}$ is inherent in the structure of $G$. We'll later see that other cycle structures in $\overleftrightarrow{G}$ can be used to construct MCB's in $G \times C_{q}$.

\section{The Diamond Space}

Our ultimate goal is to obtain MCB's of $G \times C_{q}$, where $q$ is odd and one of the factors is triangle-free. In this situation $G \times C_{q}$ is triangle-free, for if $K$ were a triangle in $G \times C_{q}$, then $\pi_{G}(K)$ and $\pi_{C_{q}}(K)$ would be a triangles in $G$ and $C_{q}$. Hence the shortest cycles in $G \times C_{q}$ have length at least four. Since-by the greedy algorithm—an MCB must contain a maximal independent set of shortest cycles, we are especially concerned with forming 
a maximal independent set of squares. This section deals with certain squares in $G \times C_{q}$ called diamonds.

If $Q=a b c$ and $R=\operatorname{def}$ are paths of length 2 in graphs $G$ and $H$, respectively, let $Q R$ denote the 4-cycle $(a, e)(b, f)(c, e)(b, d)(a, e)$ in $G \times H$, as illustrated in Figure 4. Such a subgraph $Q R$ is called a diamond in $G \times H$, and the subspace $\mathcal{D}(G \times H) \subseteq \mathcal{C}(G \times H)$ spanned by all diamonds is called the diamond space of $\mathcal{C}(G \times H)$. In what follows we construct a basis of diamonds for $\mathcal{D}\left(G \times C_{q}\right)$. Our construction involves certain spaces of paths of length 2 in the factors. Let us agree to call a path $a b c$ of length 2 in a graph a $P_{2}$ centered at $b$ in the graph. The $P_{2}$ 's of $C_{q}$ are all of form $(i-1) i(i+1)$ for $i \in \mathbb{Z}_{q}$.

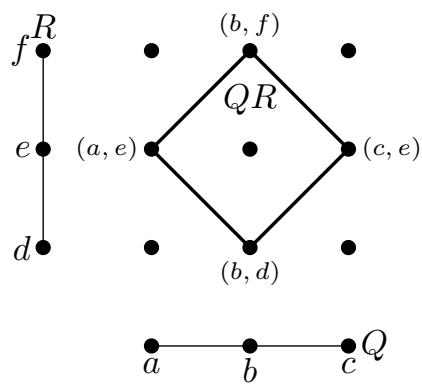

Figure 4: A diamond $Q R$

Given a vertex $b \in V(G)$, let $S(b)$ be the set of edges of $G$ that are incident with $b$. (We may think of $S(b)$ as a subgraph of $G$, i.e. the "star" at $b$.) Let $\mathcal{P}(G, b)=$ $\{X \subseteq E(S(b)):|X|$ is even $\}$. Note that $\mathcal{P}(G, b)$ is the subspace of $\mathcal{E}(S(b))$ spanned by the $P_{2}$ 's in $G$ centered at $b$, so we call it the $P_{2}$ space of $G$ at $b$. Observe that $\mathcal{P}(G, b)$ is the kernel of the surjective linear map $\mathcal{E}(S(b)) \rightarrow \mathbb{F}_{2}$ given by $X \mapsto|X|$ $(\bmod 2)$, so we have $\operatorname{dim}(\mathcal{P}(G, b))=\operatorname{dim}(\mathcal{E}(S(b)))-1=\operatorname{deg}_{G}(b)-1$. If the neighborhood of $b$ is labeled as $N_{G}(b)=\left\{x_{0}, x_{1}, x_{2}, \ldots, x_{\operatorname{deg}(b)-1}\right\}$, then the set of $P_{2}$ 's $\mathcal{B}=\left\{x_{0} b x_{1}, x_{0} b x_{2}, \ldots, x_{0} b x_{\operatorname{deg}(b)-1}\right\}$ is a basis for $\mathcal{P}(G, b)$. A basis for $\mathcal{P}(G, b)$ that consists entirely of $P_{2}$ 's is called a $P_{2}$ basis for $\mathcal{P}(G, b)$. A $P_{2}$ basis for $\mathcal{P}(G, b)$ of the form $\mathcal{B}$ above (for which some edge $x_{0} b$ belongs to every element of the basis) is called a standard $P_{2}$ basis with common edge $x_{0} b$. A version of the next lemma was proved in [9]. For completeness we include a separate proof here.

Lemma 3.1. Suppose $T$ is a tree with at least two edges, and for each $t \in V(T)$ let $\mathcal{T}_{t}$ be a $P_{2}$ basis for $\mathcal{P}(T, t)$. Then the set of diamonds $\mathcal{D}=\left\{Q[(i-1) i(i+1)]: Q \in \mathcal{T}_{t}, t \in\right.$ $\left.V(T), i \in V\left(C_{q}\right)\right\}$ is linearly independent in $\mathcal{C}\left(T \times C_{q}\right)$.

Proof. We use induction on $|E(T)|$. First suppose $|E(T)|=2$, so $T \cong P_{2}$. Label its vertices so that $T=a b c$. Then $\mathcal{D}=\left\{[a b c][(i-1) i(i+1)]: i \in V\left(C_{p}\right)\right\}$. Notice that if $i \neq j$ then diamonds $[a b c][(i-1) i(i+1)]$ and $[a b c][(j-1) j(j+1)]$ have no edges in common, since each edge in $[a b c][(i-1) i(i+1)]$ has either $(a, i)$ or $(c, i)$ as an endpoint, but no edge of $[a b c][(j-1) j(j+1)]$ has these endpoints. Thus the elements of $\mathcal{D}$ are pairwise edge-disjoint, so $\mathcal{D}$ is linearly independent.

Now suppose the statement is true for any tree $T$ with fewer than $n \geq 3$ edges, and suppose $|E(T)|=n$. For each $x \in V(T)$, let $\mathcal{T}_{x}$ be a $P_{2}$ basis for $\mathcal{P}(T, x)$. Observe 
that there is an edge of $T$ that belongs to exactly one $P_{2}$ in $\mathcal{T}=\bigcup_{x \in V(T)} \mathcal{T}_{x}$. To see this, note that since $\left|\mathcal{T}_{x}\right|=\operatorname{deg}_{T}(x)-1$ and the sets $\mathcal{T}_{x}$ are pairwise disjoint, we have $|\mathcal{T}|=$ $\sum_{x \in V(T)}\left(\operatorname{deg}_{T}(x)-1\right)=2|E(T)|-|V(T)|=|E(T)|-1$. Since each element of $\mathcal{T}$ has two edges, then on average each edge of $T$ belongs to $\frac{2(\mid E(T)-1)}{|E(T)|}<2$ elements in $\mathcal{T}$. Thus some edge of $T$ belongs to fewer than two $P_{2}$ 's in $\mathcal{T}$. But each edge in $T$ belongs to at least one element of $\mathcal{T}$, so some edge of $T$ belongs to exactly one $P_{2}$ in $\mathcal{T}$, as claimed.

Notice that if $s t$ is an edge that belongs to just one $P_{2}$ in $\mathcal{T}$, then one of $s$ or $t$ has degree 1 , for otherwise each of $\mathcal{T}_{s}$ and $\mathcal{T}_{t}$ have (distinct) elements that contains st. Thus there is an $s t \in E(T)$, with $\operatorname{deg}_{T}(s)=1$, such that $s t$ meets exactly one diamond $s t u$ in $\mathcal{T}$. Let $T^{\prime}=T-s$. (i.e. $T^{\prime}$ is $T$ with vertex $s$ and edge $s t$ removed.) Now, for each $x \in V\left(T^{\prime}\right)-\{t\}$ the set $\mathcal{T}_{x}$ remains a $P_{2}$ basis for $\mathcal{P}\left(T^{\prime}, x\right)$, and $\mathcal{T}_{t}-\{s t u\}$ is a $P_{2}$ basis for $\mathcal{P}\left(T^{\prime}, t\right)$. Let $\mathcal{T}_{x}^{\prime}=\mathcal{T}_{x}$ for $x \in V\left(T^{\prime}\right)-\{t\}$ and $\mathcal{T}_{t}^{\prime}=\mathcal{T}_{t}-\{s t u\}$.

By induction, the set $\mathcal{D}^{\prime}=\left\{Q\left[(i-1) i(i+1): Q \in \mathcal{T}_{x}^{\prime}, x \in V\left(T^{\prime}\right), i \in V\left(C_{q}\right)\right\}\right.$ is linearly independent in $\mathcal{C}\left(T^{\prime} \times C_{q}\right) \subseteq \mathcal{C}\left(T \times C_{q}\right)$. To complete the proof we need to show that the set $\mathcal{D}=\left\{Q[(i-1) i(i+1)]: Q \in \mathcal{T}_{x}, x \in V(T), i \in V\left(C_{q}\right)\right\}$ is linearly independent. Notice that $\mathcal{D}$ is a disjoint union $\mathcal{D}=\mathcal{D}^{\prime} \cup \mathcal{D}^{\prime \prime}$, where $\mathcal{D}^{\prime \prime}=\{[s t u][(i-$ 1) $\left.i(i+1)]: i \in V\left(C_{q}\right)\right\}$. By induction, the set $\mathcal{D}^{\prime \prime}$ is linearly independent in $\mathcal{C}\left(s t u \times C_{q}\right) \subseteq$ $\mathcal{C}\left(T \times C_{q}\right)$. To show that $\mathcal{D}$ is independent, it suffices to show that $\operatorname{span}\left(\mathcal{D}^{\prime}\right) \cap \operatorname{span}\left(\mathcal{D}^{\prime \prime}\right)=$ $\{0\}$. Thus suppose $W \in \operatorname{span}\left(\mathcal{D}^{\prime}\right) \cap \operatorname{span}\left(\mathcal{D}^{\prime \prime}\right)$. Notice that $\mathcal{D}^{\prime \prime}$ consists of exactly $q$ diamonds, all of form $[s t u][(i-1) i(i+1)]$, and no two of which share an edge. Thus since $W \in \operatorname{span}\left(\mathcal{D}^{\prime \prime}\right), W$ is the (possibly empty) edge-disjoint union of diamonds from $\mathcal{D}^{\prime \prime}$. But also $W \in \operatorname{span}\left(\mathcal{D}^{\prime}\right)$, so $W$ can't have any edges of form $(s, i)(t, i \pm 1)$. Since every diamond in $\mathcal{D}^{\prime \prime}$ has such edges, we conclude $W=0$.

One may wonder if the tree $T$ in Lemma 3.1 can be replaced with an arbitrary connected graph $G$. If $\mathcal{G}_{x}$ is a $P_{2}$ basis for $\mathcal{P}(G, x)$ for each $x \in V(G)$, will the set of diamonds $\mathcal{D}=$ $\left\{Q[(i-1) i(i+1)]: Q \in \mathcal{G}_{x}, x \in V(G), i \in V\left(C_{q}\right)\right\}$ be linearly independent in $\mathcal{C}\left(G \times C_{q}\right)$ ? Though the answer is "no," just a small number of diamonds need to be removed to make the set independent. The details are outlined in the following construction for a linearly independent set of diamonds in $\mathcal{D}\left(G \times C_{q}\right)$. We will only prove independence here, but later we'll see that the set is actually a basis for $\mathcal{D}\left(G \times C_{q}\right)$.

Construction 3.2. (A basis of diamonds for $\mathcal{D}\left(G \times C_{q}\right)$, with $G$ connected and $q$ odd.) Let $T$ be a spanning tree of $G$.

Let $E(G)-E(T)=\left\{b_{1} c_{1}, b_{2} c_{2}, \ldots, b_{\beta(G)} c_{\beta(G)}\right\}$.

For each $x \in V(G)$ let $\mathcal{G}_{x}$ be a standard $P_{2}$ basis for $\mathcal{P}(G, x)$ whose common edge belongs to $T$.

For each $1 \leq i \leq \beta(G)$ let $a_{i} b_{i} c_{i}$ denote the element of $\mathcal{G}_{b_{i}}$ containing the edge $b_{i} c_{i}$.

Let $\mathcal{D}=\left\{Q[(i-1) i(i+1)]: Q \in \mathcal{G}_{x}, x \in V(G), i \in V\left(C_{q}\right)\right\}-\left\{\left[a_{i} b_{i} c_{i}\right][012]: 1 \leq i \leq\right.$ $\beta(G)\}$.

Then $\mathcal{D}$ is a linearly independent set of diamonds. Moreover, $|\mathcal{D}|=\beta\left(G \times C_{q}\right)-\beta(G)-1$.

Proof. We use induction on $\beta(G)$. If $\beta(G)=0$, then $G=T$. In this case $\mathcal{D}$ is linearly independent by Lemma 3.1.

Now assume the statement is true for all $G$ with $\beta(G)<n$, for some integer $n \geq 1$. Let $G$ be a graph with $\beta(G)=n$, and let $T$ and $\mathcal{D}$ be as stated in the construction. Also, for each $1 \leq i \leq \beta(G)$, let $b_{i} c_{i} d_{i}$ denote the element of $\mathcal{G}_{c_{i}}$ containing the edge $b_{i} c_{i}$. 
Let $G^{\prime}=G-b_{\beta(G)} c_{\beta(G)}$, so $\beta\left(G^{\prime}\right)=n-1$, and $T$ is a spanning tree for $G^{\prime}$. For brevity, put $k=\beta(G)=\beta\left(G^{\prime}\right)+1$. Notice that if $x \in V(G)-\left\{b_{k}, c_{k}\right\}$, then $\mathcal{G}_{x}$ is a standard $P_{2}$ basis (with common edge in $T$ ) for both $\mathcal{P}(G, x)$ and $\mathcal{P}\left(G^{\prime}, x\right)$ because $\mathcal{P}(G, x)=$ $\mathcal{P}\left(G^{\prime}, x\right)$. Also $\mathcal{G}_{b_{k}}-\left\{a_{k} b_{k} c_{k}\right\}$ is a standard $P_{2}$ basis for $\mathcal{P}\left(G^{\prime}, b_{k}\right)$ with common edge in $T$. (Reason: $\mathcal{G}_{b_{k}}$ is a standard $P_{2}$ basis for $\mathcal{P}\left(G, b_{k}\right)$ with common edge $a_{k} b_{k}$ in $T$, so $a_{k} b_{k} c_{k}$ is the only element of $\mathcal{G}_{b_{k}}$ that contains the edge $b_{k} c_{k}$. All other members of $\mathcal{G}_{b_{k}}$ are $P_{2}$ 's in $G^{\prime}$. Since $\operatorname{dim}\left(\mathcal{P}\left(G^{\prime}, b_{k}\right)\right)=\operatorname{deg}_{G^{\prime}}\left(b_{k}\right)-1=\operatorname{deg}_{G}\left(b_{k}\right)-2=\operatorname{dim}\left(\mathcal{P}\left(G, b_{k}\right)\right)-1$, it follows that removing the single path $a_{k} b_{k} c_{k}$ from $\mathcal{G}_{b_{k}}$ must leave a basis for $\mathcal{P}\left(G^{\prime}, b_{k}\right)$.) For the same reason $\mathcal{G}_{c_{k}}-\left\{b_{k} c_{k} d_{k}\right\}$ is a standard $P_{2}$ basis for $\mathcal{P}\left(G^{\prime}, c_{k}\right)$ with common edge in $T$. Put $\mathcal{G}_{x}^{\prime}=\mathcal{G}_{x}$ if $x \in V(G)-\left\{b_{k}, c_{k}\right\}$, and $\mathcal{G}_{b_{k}}^{\prime}=\mathcal{G}_{b_{k}}-\left\{a_{k} b_{k} c_{k}\right\}$, and $\mathcal{G}_{c_{k}}^{\prime}=$ $\mathcal{G}_{c_{k}}-\left\{b_{k} c_{k} d_{k}\right\}$. Then for each $x \in V\left(G^{\prime}\right)$, the set $\mathcal{G}_{x}^{\prime}$ is a standard $P_{2}$ basis for $\mathcal{P}\left(G^{\prime}, x\right)$ with common edge in $T$.

Observe that $G^{\prime}$ meets the conditions for Construction 3.2 because $T$ is a spanning tree of $G^{\prime}$, and $E\left(G^{\prime}\right)-E(T)=\left\{b_{1} c_{1}, b_{2} c_{2}, \ldots, b_{\beta\left(G^{\prime}\right)} c_{\beta\left(G^{\prime}\right)}\right\}$, and for each $x \in V\left(G^{\prime}\right)$ the set $\mathcal{G}_{x}^{\prime}$ is a standard $P_{2}$ basis for $\mathcal{P}\left(G^{\prime}, x\right)$ whose common edge belongs to $T$, and for each $1 \leq i \leq \beta\left(G^{\prime}\right)$, the path $a_{i} b_{i} c_{i}$ is the element of $\mathcal{G}_{b_{i}}^{\prime}$ containing $b_{i} c_{i}$. By induction, the set $\mathcal{D}^{\prime}=\left\{Q[(i-1) i(i+1)]: Q \in \mathcal{G}^{\prime}{ }_{x}, x \in V\left(G^{\prime}\right), i \in V\left(C_{q}\right)\right\}-\left\{\left[a_{i} b_{i} c_{i}\right][012]: 1 \leq i \leq\right.$ $k-1\}$ is linearly independent.

Now, $\mathcal{D}$ is a disjoint union $\mathcal{D}=\mathcal{D}^{\prime} \cup \mathcal{D}^{\prime \prime}$ where $\mathcal{D}^{\prime \prime}=\{Q[(i-1) i(i+1)]: Q \in$ $\left.\left\{a_{k} b_{k} c_{k}, \quad b_{k} c_{k} d_{k}\right\}, i \in V\left(C_{q}\right)\right\}-\left\{\left[a_{k} b_{k} c_{k}\right][012]\right\}$ is a subset of the diamonds in the subgraph $\left[a_{k} b_{k} c_{k} d_{k}\right] \times C_{q}$. To show that $\mathcal{D}$ is linearly independent, we just need to show that $\mathcal{D}^{\prime \prime}$ is linearly independent and $\operatorname{span}\left(\mathcal{D}^{\prime}\right) \cap \operatorname{span}\left(\mathcal{D}^{\prime \prime}\right)=\{0\}$. This is perhaps most easily explained graphically. (Note: we cannot necessarily apply Lemma 3.1 here, for it is possible $a_{k}=d_{k}$, in which case $a_{k} b_{k} c_{k} d_{k}$ is not a tree.) Figure 5 shows the diamonds in $\mathcal{D}^{\prime \prime}$ for the case $q=9$, and the picture for the general case (with $q$ odd) is similar. Diamonds $\left[a_{k} b_{k} c_{k}\right][(i-1) i(i+1)]$ and $\left[b_{k} c_{k} d_{k}\right][(i-1) i(i+1)]$ from $\mathcal{D}^{\prime \prime}$ are petals in a flower-like arrangement, with the diamond $\left[a_{k} b_{k} c_{k}\right][012]$ missing (it does not belong to $\left.\mathcal{D}^{\prime \prime}\right)$. The shaded region covers the edges of form $\left(b_{k}, i\right)\left(c_{k}, i \pm 1\right)$ which form the set $E\left(G \times C_{q}\right)-E\left(G^{\prime} \times C_{q}\right)$.

The set $\mathcal{D}^{\prime \prime}$ is linearly independent as follows. Suppose a sum of diamonds in $\mathcal{D}^{\prime \prime}$ equals zero. Then the diamond labeled $X$ cannot occur in the sum, because the sum contains no diamond that can cancel the edge $\left(c_{k}, 1\right)\left(b_{k}, 2\right)$ of $X$. Consequently the diamond labeled $Y$ cannot occur in the sum because the sum has no term to cancel the edge $\left(b_{k}, 2\right)\left(c_{k}, 3\right)$ of $Y$. Similarly, the diamond $Z$ cannot occur in the sum because the sum has no term to cancel the edge $\left(c_{k}, 3\right)\left(b_{k}, 4\right)$. Continuing around the flower in this pattern we see that the sum has no nonzero terms, so $\mathcal{D}^{\prime \prime}$ is linearly independent.

Next we show $\operatorname{span}\left(\mathcal{D}^{\prime}\right) \cap \operatorname{span}\left(\mathcal{D}^{\prime \prime}\right)=\{0\}$. Suppose $W \in \operatorname{span}\left(\mathcal{D}^{\prime}\right) \cap \operatorname{span}\left(\mathcal{D}^{\prime \prime}\right)$. Now, the diamonds in $\mathcal{D}^{\prime}$ are subgraphs of $E\left(G^{\prime} \times C_{q}\right)$, so none of them have edges in $E\left(G \times C_{q}\right)-E\left(G^{\prime} \times C_{q}\right)$ (shaded in the figure). Thus $W$ has no edges of this form. At the same time, since $W$ is in $\operatorname{span}\left(\mathcal{D}^{\prime \prime}\right)$ it must be a sum of diamonds in $\mathcal{D}^{\prime \prime}$. Then the diamond $X$ cannot be in the sum because the sum contains no diamond that can cancel the edge $\left(c_{k}, 1\right)\left(b_{k}, 2\right)$ of $X$. Repeating the argument in the previous paragraph, the sum has no nonzero terms, so $W=0$.

This completes the proof that $\mathcal{D}$ is linearly independent. To prove the statement about 


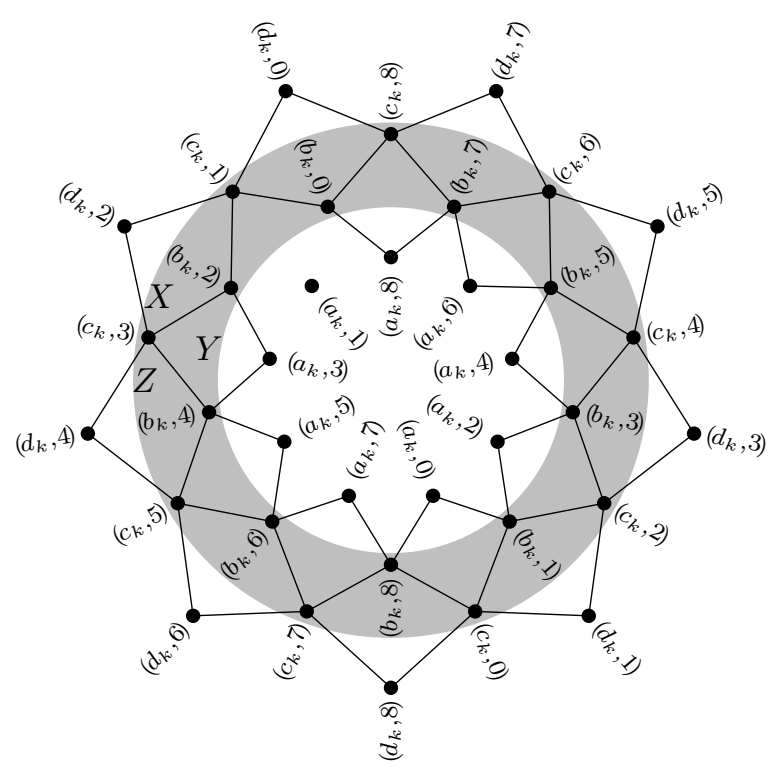

Figure 5: The set $\mathcal{D}^{\prime \prime}$

the cardinality, note that the definition of $\mathcal{D}$ yields

$$
\begin{aligned}
|\mathcal{D}|= & \left|\left\{Q[(i-1) i(i+1)]: Q \in \mathcal{G}_{x}, x \in V(G), i \in V\left(C_{q}\right)\right\}\right|- \\
& \left|\left\{\left[a_{i} b_{i} c_{i}\right][012]: 1 \leq i \leq \beta(G)\right\}\right| \\
= & \sum_{x \in V(G)}\left|\mathcal{G}_{x}\right| q-\beta(G) \\
= & q\left(\sum_{x \in V(G)} \operatorname{deg}_{G}(x)-1\right)-(|E(G)|-|V(G)|+1) \\
= & q(2|E(G)|-|V(G)|)-(|E(G)|-|V(G)|+1) \\
= & (2|E(G)| q-|V(G)| q+1)-(|E(G)|-|V(G)|+1)-1 \\
= & \beta\left(G \times C_{q}\right)-\beta(G)-1 .
\end{aligned}
$$

The proof is now complete.

Before moving on, we visit a consequence of Construction 3.2 that will be useful.

Lemma 3.3. Suppose $G$ is a connected non-bipartite graph, e is an edge of $C_{q}$ and $P$ is the path $C_{q}-e$. Let $a b \in E(P)$, and regard $G \times a b \cong G \times K_{2}$ as a subgraph of $G \times P$. Then $\mathcal{C}(G \times P)=\mathcal{C}(G \times a b) \bigoplus \mathcal{D}(G \times P)$. Moreover, if $C=A+B$ with $A \in \mathcal{C}(G \times a b)$ and $B \in \mathcal{D}(G \times P)$, then $|C| \geq|A|$.

Proof. Let the partite sets of $P$ be $X_{a}$ and $X_{b}$, with $a \in X_{a}$ and $b \in X_{b}$. Observe that there is a homomorphism $\rho: G \times P \rightarrow G \times a b$ defined as $\rho(x, i)=(x, a)$ if $i \in X_{a}$ 
and $\rho(x, i)=(x, b)$ if $i \in X_{b}$. Note that $\rho$ is the identity on $G \times a b$, and it thus induces a projection $\rho^{*}: \mathcal{C}(G \times P) \rightarrow \mathcal{C}(G \times a b)$. Therefore $\mathcal{C}(G \times P)=\mathcal{C}(G \times a b) \bigoplus \operatorname{ker}\left(\rho^{*}\right)$, so $\operatorname{dim}\left(\operatorname{ker}\left(\rho^{*}\right)\right)=\beta(G \times P)-\beta(G \times a b)$. Now $\rho^{*}(D)=0$ for any diamond $D$, so $\mathcal{D}(G \times P) \subseteq \operatorname{ker}\left(\rho^{*}\right)$. We will finish the proof by producing a linearly independent set of diamonds in $\mathcal{D}(G \times P)$ of cardinality $\beta(G \times P)-\beta(G \times a b)$.

We can create an independent set of diamonds in $\mathcal{D}(G \times P)$ by removing from the set $\mathcal{D}$ of Construction 3.2 all diamonds that are not in $G \times P$. If $e=c(c+1)$, we remove the diamonds of form $Q[(c-1) c(c+1)]$ and $Q[c(c+1)(c+2)]$ and obtain the independent set $\mathcal{B}=\left\{Q[(i-1) i(i+1)]: Q \in \mathcal{G}_{x}, x \in V(G), i \in \mathbb{Z}_{q}-\{c, c+1\}\right\}$ of diamonds in $G \times P$. Observe

$$
\begin{aligned}
|\mathcal{B}| & =\sum_{x \in V(G)}\left|\mathcal{G}_{x}\right|(q-2)=(q-2) \sum_{x \in V(G)}\left(\operatorname{deg}_{G}(x)-1\right) \\
& =(q-2)(2|E(G)|-|V(G)|) \\
& =(2|E(G)|(q-1)-V(G) \mid q+1)-(2|E(G)|-2|V(G)|+1) \\
& =\beta(G \times P)-\beta(G \times a b) .
\end{aligned}
$$

Finally, suppose $C=A+B$ as in the statement of the lemma. Now, $|C| \geq\left|\rho^{*}(C)\right|$ because any edge in $\rho^{*}(C)$ must be the image under $\rho^{*}$ of at least one edge in $C$. Since $\rho^{*}(C)=\rho^{*}(A+B)=\rho^{*}(A)+\rho^{*}(B)=\rho^{*}(A)=A$, we have $|C| \geq|A|$.

\section{The Product of Two Odd Cycles}

In this section we take up the problem of finding an MCB for the product of cycles $C_{p} \times C_{q}$ where $p$ and $q$ are odd and at least one is greater than 3 . For simplicity assume $p \leq q$. This is a special case of our ultimate problem of finding an MCB of $G \times C_{q}$, but treating it now will help us understand some of the subtleties of the general problem and put us in a position to better understand and motivate our general construction. The discussion is informal and is intended for illumination only, and any lack of rigor will be compensated in the subsequent section. Though the arguments used in this section are topological, those that follow will be entirely combinatorial.

Observe that $C_{p} \times C_{q}$ can be embedded on the torus with $p q$ square regions whose boundaries are the diamonds of $C_{p} \times C_{q}$. This is illustrated for $C_{5} \times C_{9}$ in Figure 6(a) and for $C_{5} \times C_{11}$ in Figure 6(b). In each case the torus is an identification space obtained by identifying paths $A$ (of length $p$ ), paths $B$ (of length $p$ ), and the zig-zag path $C$ (of length $q-p$ ). The general case is illustrated in figures 7(a) and 7(b).

The set of all $p q$ diamonds is linearly dependent, for if they are all added together their edges will cancel pair-by-pair. But set $\mathcal{D}$ of Construction 3.2 is independent and $|\mathcal{D}|=$ $\beta\left(C_{p} \times C_{q}\right)-\beta\left(C_{q}\right)-1=(2 p q-p q+1)-1-1=p q-1$. Thus $\mathcal{D}$ contains all but one diamond, and the missing one is the sum everything in $\mathcal{D}$, so $\mathcal{D}$ is a basis for $\mathcal{D}\left(C_{p} \times C_{q}\right)$.

Let's now use the Greedy Algorithm to obtain an MCB. There are no cycles of length less than 4 , so begin by setting $\mathcal{M}:=\mathcal{D}$. Since $\beta\left(C_{p} \times C_{q}\right)=p q+1=|\mathcal{M}|+2$, there are just two more cycles to append.

As an aid in finding these two cycles, we claim any even cycle $Z \in \mathcal{C}\left(C_{p} \times C_{q}\right)$ with $|Z|<2 p$ is a sum of diamonds, and is thus already in $\operatorname{span}(\mathcal{M})$. For if $Z$ is such an even cycle, the homomorphism $\pi_{C_{p}}^{*}: \mathcal{C}\left(C_{p} \times C_{q}\right) \rightarrow \mathcal{C}\left(C_{p}\right)=\left\{0, C_{p}\right\}$ must send $Z$ to an even cycle, so $\pi_{C_{p}}^{*}(Z)=0$. Then for any edge $e=a b \in E\left(C_{p}\right)$, cycle $Z$ must have 


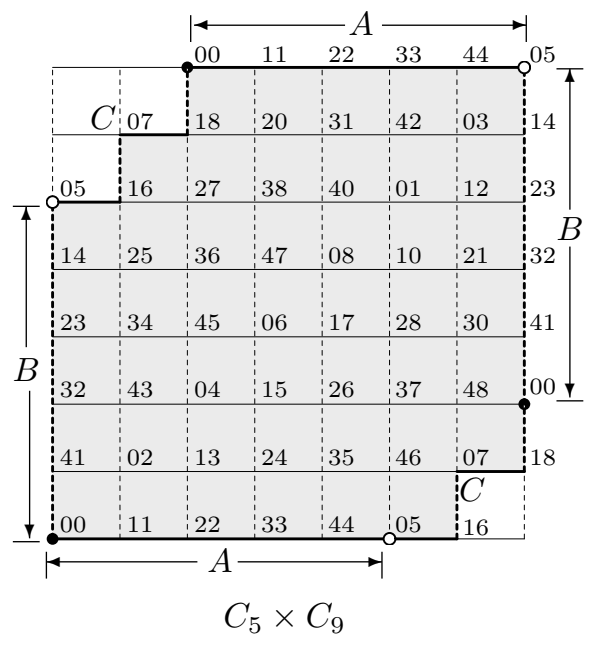

(a)

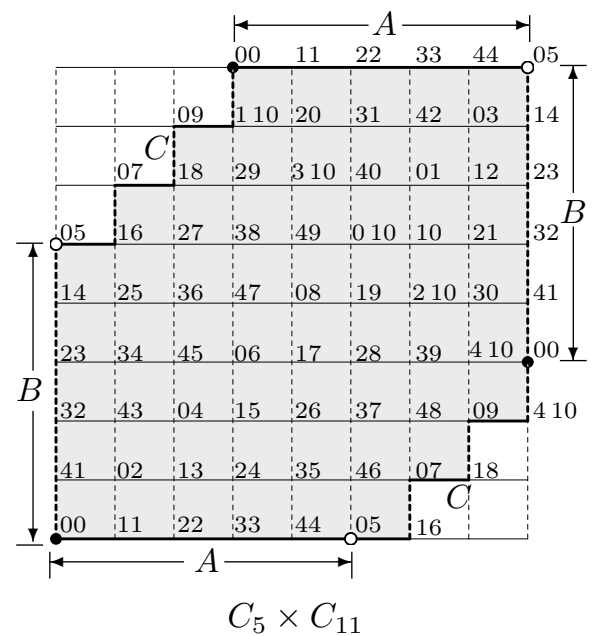

(b)

Figure 6: The graph $C_{p} \times C_{q}$ on the torus

an even number $m_{e}$ of edges of form $(a, x)(b, y)$ for which $\pi_{C_{p}}((a, x)(b, y))=a b$. Since $2 p>|Z|=\sum_{e \in E\left(C_{p}\right)} m_{e}$, it follows that $m_{e}=0$ for some $e \in E\left(C_{p}\right)$. Hence $Z$ is a cycle in the graph $\left(C_{p}-e\right) \times C_{q}$. By applying the same argument to the factor $C_{q}$ (and using $p \leq q$ ) we see $C_{q}$ must have some edge $f$ for which $Z$ is a cycle in the product $\left(C_{p}-e\right) \times\left(C_{q}-f\right)$ of paths. A product of paths has two planar components which can be embedded in the plane so that the boundaries of all interior regions are diamonds. By MacLane's theorem, these diamonds span the cycle space, so $Z$ is a sum of diamonds.

In Figure 6 the edges of the products are colored solid and dashed according to whether they run horizontally or vertically in the grids. With this coloring, every diamond has two edges of each color, so any element of $\operatorname{span}(\mathcal{M})$ has an even number of edges of each color. Now the even cycle $A+B$ of length $2 p$ has $p$ (odd) edges of each color, $A+B \notin$ $\operatorname{span}(\mathcal{M})$. Further, $A+C$ and $B+C$ are cycles of length $q$ (odd), so they are certainly not in $\operatorname{span}(\mathcal{M})$. Since $A+B=(A+C)+(B+C)$, it follows that appending to $\mathcal{M}$ any two elements of $\{A+B, A+C, B+C\}$ will produce a basis.

Now continue with the Greedy Algorithm. We know that if an even cycle is appended to $\mathcal{M}$, the even cycle must have length no less than $2 p$. At the same time, since $C_{p} \times C_{q}$ has odd cycles, at least one odd cycle must appear in an $\mathrm{MCB}$, and such an odd cycle can have length no less than $q$. Therefore if $q<2 p$, then $\mathcal{M}$ can be extended to an MCB by appending to it the odd cycles $A+C$ and $B+C$ of length $q$. On the other hand, if $2 p<q$, then $\mathcal{M}$ is extended to an MCB by appending to it the even cycle $A+B$ of length $2 p$ the odd cycle $A+C$ of length $q$. This proves the following result.

Proposition 4.1. Suppose $p$ and $q$ are odd integers, $p \leq q$ and $\max \{p, q\}>3$.

If $q<2 p$, then $C_{p} \times C_{q}$ has an MCB consisting of $p q-1$ squares and two $q$-cycles. If $2 p<q$, then $C_{p} \times C_{q}$ has an MCB consisting of $p q-1$ squares, a $2 p$-cycle and a $q$-cycle. 


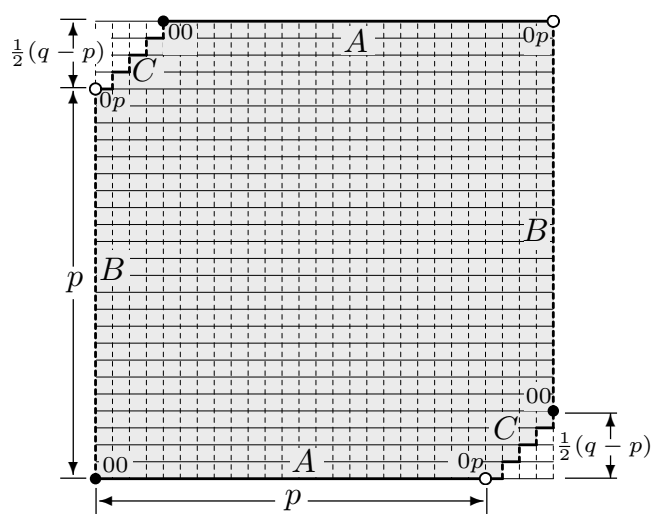

(a)

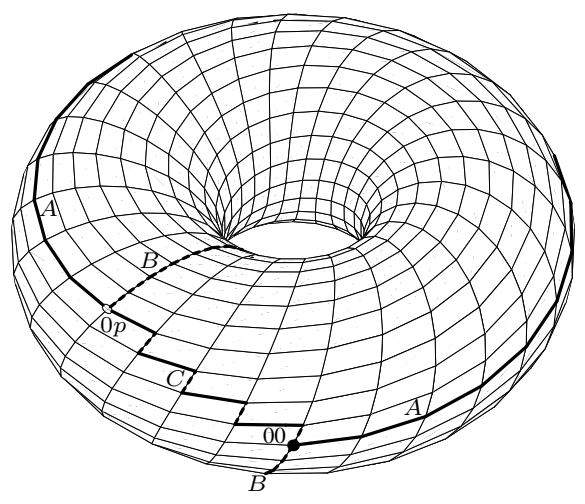

(b)

Figure 7: The graph $C_{p} \times C_{q}$ on the torus

Figures 6(a) and 6(b) illustrate this proposition. In Figure 6(a) $C_{5} \times C_{9}$ has an MCB consisting of 44 diamonds, and two 9-cycles $A+C$ and $B+C$. In Figure 6(a) $C_{5} \times C_{11}$ has an MCB consisting of 54 diamonds, one 10-cycle $A+B$ and one 11-cycle $A+C$.

\section{An MCB for $G \times C_{q}$}

In the previous section we constructed an MCB for $C_{p} \times C_{q}$ where $p, q$ are odd, $p \leq q$ and $\max \{p, q\}>3$. We now generalize this by replacing the factor $C_{p}$ with a connected graph $G$ whose shortest odd cycle has length $p$. That is, we construct an MCB for $G \times C_{q}$ where $p \leq q$ and $\max \{p, q\}>3$. Under these hypotheses every odd cycle in $G \times C_{q}$ has length at least $q$, so $G \times C_{q}$ is triangle-free.

As was the case for $G \times K_{2}$ in Section 2, we should not expect an MCB of $G \times C_{q}$ to correspond in any way to an MCB of $G$. And as in Section 2 our approach here will be to replace $G$ with its symmetric digraph $\overleftrightarrow{G}$ and transfer minimal cycle structures of $\overleftrightarrow{G}$ to $G \times C_{q}$. However, since $G \times C_{q}$ has odd cycles (unlike $G \times K_{2}$ ), the anti-cycle space $\mathcal{A}(\overleftrightarrow{G})$ (whose elements all possess an even number of arcs) does not have an adequate cycle structure for the job at hand, and we will have to enlarge it slightly. The relevant definitions follow.

As in Section 2, let $\overleftrightarrow{G}$ denote the symmetric digraph on $G$ with arc set $E(\overleftrightarrow{G})=$ $\{\overrightarrow{x y}, \overrightarrow{y x}: x y \in E(G)\}$. A pair $\{\overrightarrow{x y}, \overrightarrow{y x}\}$ is called a double edge of $\overleftrightarrow{G}$. A sub-digraph of $\overleftrightarrow{G}$ is symmetric if whenever $\overrightarrow{x y}$ is one of its arcs, then $\overrightarrow{y x}$ is also one of its arcs (i.e. if every one of its arcs is part of a double edge.)

Let $\mathcal{C}(\overleftrightarrow{G})$ be the kernel of the linear map $\lambda: \mathcal{E}(\overleftrightarrow{G}) \rightarrow \mathcal{V}(G)$ defined as $\lambda(\overrightarrow{x y})=$ $\{x\}+\{y\}$. One easily checks that $\mathcal{C}(\overleftrightarrow{G})$ consists of the edge sets of the sub-digraphs of $\overleftrightarrow{G}$ for which at each vertex the sum of the in- and out-degree is even. Observe that $\mathcal{A}(\overleftrightarrow{G})$ is a proper subspace of $\mathcal{C}(\overleftrightarrow{G})$, provided that $G$ has at least one edge. Indeed, any double edge $\{\overrightarrow{x y}, \overrightarrow{y x}\} \in \mathcal{E}(\overleftrightarrow{G})$ is in $\mathcal{C}(\overleftrightarrow{G})$ but not in $\mathcal{A}(\overleftrightarrow{G})$. The range of $\lambda$ is 
the subspace of $\{X \subset V(G):|X|$ is even $\}$ of $\mathcal{V}(G)$, and its dimension is $|V(G)|-1$. (Because it is the kernel of the surjective linear map $\mathcal{V}(G) \rightarrow \mathbb{F}_{2}$ defined as $X \rightarrow|X|$ $(\bmod 2)$.) Therefore, since $\operatorname{rank}(\lambda)+\operatorname{dim}(\operatorname{ker}(\lambda))=\operatorname{dim}(\mathcal{E}(\overleftrightarrow{G}))=2|E(G)|$, we see $\operatorname{dim}(\mathcal{C}(\overleftrightarrow{G}))=2|E(G)|-|V(G)|+1$

Just as we regard elements of $\mathcal{C}(G)$ as (eulerian) subgraphs of $G$, we regard elements of $\mathcal{C}(\overleftrightarrow{G})$ as the sub-digraphs of $\overleftrightarrow{G}$ for which the total degree (in-degree plus out-degree) of each vertex is even. Recall that an orientation of a graph is an assignment of a direction to each of its edges. Thus if $A \in \mathcal{C}(G)$, any orientation of $A$ is in $\mathcal{C}(\overleftrightarrow{G})$. However an orientation of $A$ has no double edges, while an element of $\mathcal{C}(\overleftrightarrow{G})$ may have double edges. Consequently though $\mathcal{C}(\overleftrightarrow{G})$ contains all the orientations of eulerian subgraphs of $G$, not every element of $\mathcal{C}(\overleftrightarrow{G})$ is such an orientation.

We now define a projection $\pi: \mathcal{C}\left(G \times C_{q}\right) \rightarrow \mathcal{C}(\overleftrightarrow{G})$. Consider the linear map $\pi:$ $\mathcal{E}\left(G \times C_{q}\right) \rightarrow \mathcal{E}(\overleftrightarrow{G})$ which acts on the basis $E\left(G \times C_{q}\right)$ as

$$
\pi((v, i)(w, j))= \begin{cases}\overrightarrow{v w} & \text { if } j=i+1 \\ \overrightarrow{w v} & \text { if } j=i-1\end{cases}
$$

It is straightforward to check that this restricts to a linear map $\pi: \mathcal{C}\left(G \times C_{q}\right) \rightarrow \mathcal{C}(\overleftrightarrow{G})$

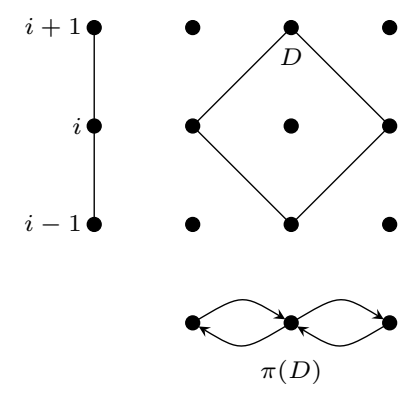

Figure 8: Projection of a diamond

As an example, observe that (as illustrated in Figure 8) if $D$ is a diamond, then $\pi(D)$ consists of two double edges. It follows that if $Y \in \mathcal{D}\left(G \times C_{q}\right)$, then $\pi(Y)$ consists of an even number of double edges.

Our reason for constructing $\mathcal{C}(\overleftrightarrow{G})$ is to have a richer version of $\mathcal{C}(G)$ so that, roughly, we may ultimately be able to lift some minimal cycle structure in $\mathcal{C}(\overleftrightarrow{G})$ to $\mathcal{C}\left(G \times C_{q}\right)$. We've seen that if $Y \in \mathcal{D}\left(G \times C_{q}\right)$, then $\pi(Y)$ is a symmetric sub-digraph of $\overleftrightarrow{G}$ that consists of an even number of double edges. By Construction 3.2 we already have a set $\mathcal{D}$ of diamonds that spans $\mathcal{D}\left(G \times C_{q}\right)$, so we are not interested in lifting such sub-digraphs $Y$ to cycles in $\mathcal{D}\left(G \times C_{q}\right)$. Thus we next cut down the size of $\mathcal{C}(\overleftrightarrow{G})$ by forming the quotient of if with the space of symmetric digraphs with an even number of double edges.

Let $\mathcal{V}=\{Y \in \mathcal{C}(\overleftrightarrow{G}): Y$ is symmetric and $|Y| \equiv 0(\bmod 4)\}$. Note $\mathcal{V}$ is precisely the subset of $\mathcal{C}(\overleftrightarrow{G})$ whose elements are symmetric digraphs with an even number of double edges. (Each double edge contains two opposing arcs, so an even number of double edges 
yields a total number of arcs that is a multiple of 4.) Clearly $\mathcal{V}$ is a subspace of $\mathcal{C}(\overleftrightarrow{G})$. If we identify the double edges in $\overleftrightarrow{G}$ with edges in $G$, then $\mathcal{V}$ is identified with the subspace $\mathcal{W}=\{X \subseteq E(G):|X|$ is even $\}$ of $\mathcal{E}(G)$. Since $\operatorname{dim}(\mathcal{W})=|E(G)|-1$, we obtain $\operatorname{dim}(\mathcal{V})=|E(G)|-1$.

Now consider the quotient $\mathcal{C}(\overleftrightarrow{G}) / \mathcal{V}$. The dimension of this space is $\operatorname{dim}(\mathcal{C}(\overleftrightarrow{G}))-$ $\operatorname{dim}(\mathcal{V})=(2|E(G)|-|V(G)|+1)-(|E(G)|-1)=\beta(G)+1$. Since its dimension is just one more than the dimension of $\mathcal{C}(G)$, we would expect the structure of $\mathcal{C}(\overleftrightarrow{G}) / \mathcal{V}$ to be similar to - but slightly richer than - the structure of $\mathcal{C}(G)$. In fact we will soon see that elements of $\mathcal{C}(\overleftrightarrow{G}) / \mathcal{V}$ can be lifted to part of an MCB for $G \times C_{q}$, whereas that is not necessarily possible for lifts of elements of $\mathcal{C}(G)$.

Example 5.1. As a specific example of $\mathcal{C}(\overleftrightarrow{G}) / \mathcal{V}$, consider the case $G=C_{p}$, where $p$ is odd. Note that $\mathcal{C}(\overleftrightarrow{G}) / \mathcal{V}$ has dimension $\beta(G)+1=2$. Let $A_{1}$ be the digraph obtained from $C_{p}$ by giving it the orientation where each arc is directed from $i$ to $i+1$. Let $A_{2}$ be $A_{1}$ with arcs reversed, that is the arcs in $A_{2}$ are directed from $i$ to $i-1$. Since neither $A_{1}$ nor $A_{2}$ is symmetric, $A_{1}+\mathcal{V}$ and $A_{2}+\mathcal{V}$ are nonzero in $\mathcal{C}(\overleftrightarrow{G}) / \mathcal{V}$. Also $A_{1}+A_{2}$ is a symmetric digraph with $p$ (odd) double edges, so $A_{1}+A_{2} \notin \mathcal{V}$ It follows that the set $\left\{A_{1}+\mathcal{V}, A_{2}+\mathcal{V}\right\}$ is linearly independent in $\mathcal{C}(\overleftrightarrow{G}) / \mathcal{V}$. Thus $\mathcal{C}(\overleftrightarrow{G}) / \mathcal{V}=\left\{\mathcal{V}, A_{1}+\mathcal{V}, A_{2}+\mathcal{V},\left(A_{1}+A_{2}\right)+\mathcal{V}\right\}$

Definition 5.2. Let $\vec{C}_{n}$ be the digraph with vertices $\mathbb{Z}_{n}$ and with arcs directed from $i$ to $i+1$ for each $i \in \mathbb{Z}_{n}$. A sub-digraph $C$ in $\overleftrightarrow{G}$ is called a directed cycle if it is isomorphic to $\vec{C}_{n}$.

In the next lemma we need the linear function $f: \mathcal{E}(\overleftrightarrow{G}) \rightarrow \mathcal{E}(G)$ defined on basis $E(\overleftrightarrow{G})$ as $f(\overrightarrow{x y})=x y$. Thus $f$ simply eliminates all double edges of its argument and "forgets" the orientation of the remaining arcs. The kernel of $f$ is the space of symmetric digraphs in $\mathcal{E}(\overleftrightarrow{G})$. It is easy to check that $f$ restricts to a map $f: \mathcal{C}(\overleftrightarrow{G}) \rightarrow \mathcal{C}(G)$

Lemma 5.3. If $G$ is non-bipartite, then $\mathcal{C}(\overleftrightarrow{G}) / \mathcal{V}$ is spanned by the elements $A+\mathcal{V}$ where $A$ is a directed odd cycle or an anti-cycle.

Proof. Let $\mathcal{A}=\left\{A_{1}, A_{2}, \ldots, A_{\beta(G)}\right\}$ be a basis of $\mathcal{C}(G)$ consisting of simple cycles (an MCB will suffice). For each index $i$, give $A_{i}$ an orientation that makes it an anti-cycle if $\left|A_{i}\right|$ is even, or a directed cycle if $\left|A_{i}\right|$ is odd. Call the resulting digraph $A_{i}^{\prime}$. Thus we have $f\left(A_{i}^{\prime}\right)=A_{i}$. The set $\mathcal{A}^{\prime}=\left\{A_{1}^{\prime}+\mathcal{V}, A_{2}^{\prime}+\mathcal{V}, \ldots, A_{\beta(G)}^{\prime}+\mathcal{V}\right\}$ is linearly independent in $\mathcal{C}(\overleftrightarrow{G}) / \mathcal{V}$ for the following reason. Suppose $\sum_{i \in I}\left(A_{i}^{\prime}+\mathcal{V}\right)=\mathcal{V}$, where $I \subseteq\{1,2, \ldots, \beta(G)\}$. Then $\sum_{i \in I} A_{i}^{\prime} \in \mathcal{V}$. Since any element of $\mathcal{V}$ is symmetric we have $f\left(\sum_{i \in I} A_{i}^{\prime}\right)=\sum_{i \in I} A_{i}=0$. Thus $I=\emptyset$, showing $\mathcal{A}^{\prime}$ is independent.

Now let $A_{\beta(G)+1}^{\prime}$ be the symmetric digraph on a simple odd cycle of $G$. (That is it is obtained by replacing each edge of a simple odd cycle of $G$ with a double edge.) Then $A_{\beta(G)+1}^{\prime}$ is an anti-cycle. Now even though $A_{\beta(G)+1}^{\prime}$ is symmetric, it has an odd number of double edges so $A_{\beta(G)+1}^{\prime}+\mathcal{V}$ is nonzero in $\mathcal{C}(\overleftrightarrow{G}) / \mathcal{V}$. We now show that it is not a linear combination of elements in $\mathcal{A}^{\prime}$. Suppose to the contrary that $A_{\beta(G)+1}^{\prime}+\mathcal{V}=$ $\sum_{i \in I}\left(A_{i}^{\prime}+\mathcal{V}\right)$. Then $A_{\beta(G)+1}^{\prime}+\mathcal{V}=\left(\sum_{i \in I} A_{i}^{\prime}\right)+\mathcal{V}$, so $\sum_{i \in I} A_{i}^{\prime}$ is symmetric, as it is the 
sum of the symmetric digraph $A_{\beta(G)+1}^{\prime}$ and a symmetric graph in $\mathcal{V}$. Applying $f$ we have $\sum_{i \in I} A_{i}=0$, a contradiction.

Thus we have linearly independent set $\left\{A_{1}^{\prime}+\mathcal{V}, A_{2}^{\prime}+\mathcal{V}, \ldots, A_{\beta(G)}^{\prime}+\mathcal{V}, A_{\beta(G)+1}^{\prime}+\mathcal{V}\right\}$ with each $A_{i}^{\prime}$ is an anticycle or a directed odd cycle. Since $\mathcal{C}(\overleftrightarrow{G}) / \mathcal{V}$ is known to have dimension $\beta(G)+1$, we are done.

If $\widetilde{A} \in \mathcal{C}\left(G \times C_{q}\right)$ and $\pi(\widetilde{A})=A$, then certainly $|\widetilde{A}| \geq|A|$ because each arc in $A$ is the projection of at least one edge in $\widetilde{A}$. Moreover $|A|$ is odd if and only if $|\widetilde{A}|$ is odd, and in such cases $|\widetilde{A}| \geq \max \{q,|A|\}$ because $G \times C_{q}$ has no odd cycles of length less than $q$. The next lemmas show that if $A$ is an anti-cycle or a directed odd cycle, then there is some $\widetilde{A} \in \mathcal{C}\left(G \times C_{q}\right)$ for which $\pi(\widetilde{A})=A$ (modulo $\mathcal{V}$ ), and for which $\widetilde{A}$ attains the minimum length $|A|$ if $A$ is an anti-cycle, or $\max \{q,|A|\}$ if $A$ is a directed odd cycle. Let $\pi^{\prime}: \mathcal{C}\left(G \times C_{q}\right) \rightarrow \mathcal{C}(\overleftrightarrow{G}) / \mathcal{V}$ be the map $\pi^{\prime}(C)=\pi(C)+\mathcal{V}$

Lemma 5.4. If $A$ is an anti-cycle in $\mathcal{C}(\overleftrightarrow{G})$, then there is a cycle $\widetilde{A} \in \mathcal{C}\left(G \times C_{q}\right)$ with $|\widetilde{A}|=|A|$ and $\pi(\widetilde{A})=A$. In particular $\pi^{\prime}(\widetilde{A})=A+\mathcal{V}$.

Proof. Given anti-cycle $A$, let $\widetilde{A}=\{(x, 0)(y, 1): \overrightarrow{x y} \in E(A)\}$, as illustrated in Figure 3 . By construction $|\widetilde{A}|=|A|$ and $\pi(\widetilde{A})=A$.

Lemma 5.5. If $A$ is a directed odd cycle in $\mathcal{C}(\overleftrightarrow{G})$, then there is a cycle $\widetilde{A} \in \mathcal{C}\left(G \times C_{q}\right)$ with $|\widetilde{A}|=\max \{q,|A|\}$ and $\pi^{\prime}(\widetilde{A})=A+\mathcal{V}$.

Proof. Say $A$ has $n$ vertices. Label its vertices with the elements of $\mathbb{Z}_{n}$ so that each arc of $A$ has form $\overrightarrow{i(i+1)}$. We consider four cases.

Case (a). Suppose $q>n$ and $q-n \equiv 0(\bmod 4)$. Let $\widetilde{A}$ be the concatenation of paths

$$
\begin{aligned}
L & =(0,0)(1,1)(2,2) \ldots(n-1, n-1)(0, n) \\
\text { and } \quad M & =(0, n)(1, n+1)(0, n+2)(1, n+3) \ldots(0,0)
\end{aligned}
$$

of lengths $n$ and $q-n$ respectively, which are shown solid and dashed in Figure 9(a). Then $|\widetilde{A}|=q=\max \{q,|A|\}$. Further, $\pi(L)=A$. Moreover, $\pi$ sends every two successive edges of $M$ to $\overrightarrow{01}+\overrightarrow{10}$. Since the length of $M$ is a multiple of 4 , it follows that $\pi(M)=0$. Therefore $\pi(\widetilde{A})=\pi(L)+\pi(M)=A+0=A$, so $\pi^{\prime}(\widetilde{A})=A+\mathcal{V}$, as required.

Case (b). Suppose $q>n$ and $q-n \equiv 2(\bmod 4)$. If we used $L$ and $M$ from the previous case, then $\pi(L)=\overrightarrow{01}+\overrightarrow{10}$, so $\pi(\widetilde{A})$ would be $A$ with the arc 01 replaced with 10 . Instead, let $\widetilde{A}$ be the concatenation of

$$
\begin{aligned}
L & =(0,0)(n-1,1)(n-2,2) \ldots(1, n-1)(0, n) \\
\text { and } \quad M & =(0, n)(1, n+1)(0, n+2)(1, n+3) \ldots(0,0)
\end{aligned}
$$

which are shown solid and dashed, respectively in Figure 9(b). Then $\pi(L)$ is the reverse orientation of $\mathrm{A}$, so $\pi(L)+A$ is a symmetric graph with $n$ (odd) double edges. Also $\pi(M)=\overrightarrow{01}+\overrightarrow{10}$, so $\pi(L)+\pi(M)+A=\pi(\widetilde{A})+A$ is a symmetric graph with $n-1$ (even) double edges. Hence $\pi(\widetilde{A})+A \in \mathcal{V}$, so $\pi^{\prime}(\widetilde{A})=A+\mathcal{V}$, as required. 
Case (c). Suppose $q \leq n$ and $n-q \equiv 0(\bmod 4)$. Let $\widetilde{A}$ be the concatenation of paths

$$
\begin{aligned}
L & =(0,0)(1,1)(2,2)(3,3) \ldots(q, 0) \\
\text { and } \quad M & =(q, 0)(q+1,1)(q+2,0)(q+3,1)(q+4,0) \ldots(n-1,1)(0,0)
\end{aligned}
$$

of lengths $q$ and $n-q$, which are shown solid and dashed, respectively in Figure 9(c). Notice that $|\widetilde{A}|=|A|=\max \{q,|A|\}$. Also $\pi(\widetilde{A})=\pi(L)+\pi(M)$ is the directed graph obtained from $A$ by reversing every other arc in the directed path $q(q+1)(q+2) \ldots 0$ in $A$. Since the number of arcs in this path is a multiple of $4, \pi(\widetilde{A})$ is just $A$ with an even number of arcs reversed. It follows that $\pi(\widetilde{A})+A$ is a symmetric graph with an even number of double edges, so $\pi(\widetilde{A})+A \in \mathcal{V}$, hence $\pi^{\prime}(\widetilde{A})=A+\mathcal{V}$.

Case (d). Suppose $q \leq n$ and $n-q \equiv 2(\bmod 4)$. Let $\widetilde{A}$ be the concatenation of paths

$$
\begin{aligned}
L & =(0,0)(n-1,1)(n-2,2)(n-3,3) \ldots(n-q, 0) \quad \text { and } \\
M & =(n-q, 0)(n-q+1,1)(n-q+2,0)(q+3,1)(n-q+4,0) \ldots(n-1,1)(0,0)
\end{aligned}
$$

of lengths $q$ and $n-q$ and reason as above.

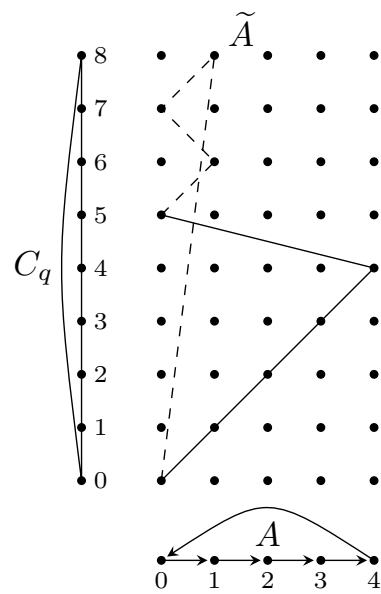

(a)

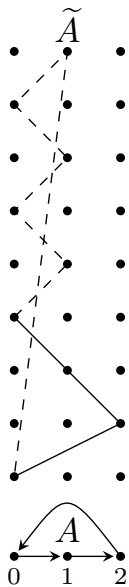

(b)

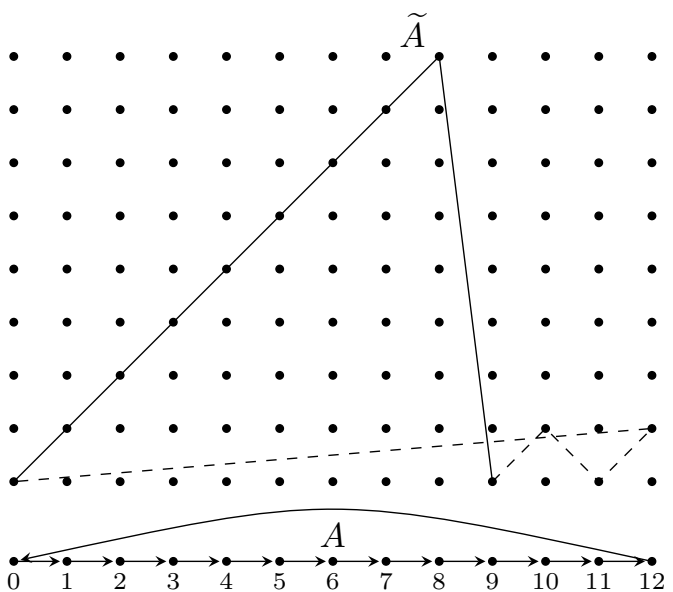

(c)

Figure 9: Lifts of directed odd cycles

In order to lift a minimum cycle structure of $\mathcal{C}(\overleftrightarrow{G}) / \mathcal{V}$ to part of an MCB of $G \times C_{q}$ it will be necessary to weight elements $A+\mathcal{V}$ not by $|A|$, but by the number of edges in a lift of $A$. Hence the following definition, motivated by the previous two lemmas.

Definition 5.6. If $A \in \mathcal{C}(\overleftrightarrow{G})$ is an anti-cycle or a directed odd cycle then its $q$-weight is the integer

$$
w_{q}(A)=\left\{\begin{array}{cl}
|A| & \text { if } A \text { is an anti-cycle } \\
\max \{q,|A|\} & \text { if } A \text { is a directed odd cycle. }
\end{array}\right.
$$

A basis $\mathcal{A}=\left\{A_{1}+\mathcal{V}, A_{2}+\mathcal{V}, \ldots, A_{\beta(G)+1}+\mathcal{V}\right\}$ for $\mathcal{C}(\overleftrightarrow{G}) / \mathcal{V}$ is called a minimum $q$-weight basis if each $A_{i}$ is an anti-cycle or a directed odd cycle and the total $q$-weight 
$\sum_{i=1}^{\beta(G)+1} w_{q}\left(A_{i}\right)$ has the minimum possible value among all such bases. (A minimum $q$-weight basis exists by Lemma 5.3.)

We can finally state our construction for an MCB of $G \times C_{q}$.

Construction 5.7. (An MCB for $G \times C_{q}$ where $G$ is connected and non-bipartite, $q$ is odd, the shortest odd cycle in $G$ has length $p \leq q$, and $\max \{p, q\}>3$.)

1. Let $\mathcal{D}$ be the set of diamonds from Construction 3.2.

2. Let $\mathcal{A}=\left\{A_{1}+\mathcal{V}, A_{2}+\mathcal{V}, \ldots, A_{\beta(G)+1}+\mathcal{V}\right\}$ be a minimum q-weight basis for $\mathcal{C}(\overleftrightarrow{G}) / \mathcal{V}$

3. Let $\widetilde{\mathcal{A}}=\left\{\widetilde{A}_{1}, \widetilde{A}_{2}, \ldots, \widetilde{A}_{\beta(G)+1}\right\} \subseteq \mathcal{C}\left(G \times C_{q}\right)$ be such that $\pi\left(\widetilde{A}_{i}\right)+\mathcal{V}=A_{i}+\mathcal{V}$ and $\left|\widetilde{A}_{i}\right|=w_{q}\left(A_{i}\right)$ for each index $1 \leq i \leq \beta(G)+1$. (As in Lemmas 5.4 and 5.5.)

Then $\mathcal{B}=\widetilde{\mathcal{A}} \cup \mathcal{D}$ is an $M C B$ for $G \times C_{q}$.

Before proving this, let's look at a simple example.

Example 5.8. Consider the case of constructing an MCB of $G \times C_{q}$ where $G=C_{p}$, which was addressed in Section 4. Assume, as we did in that section, that $p \leq q$ and $\max \{p, q\}>$ 3. In Step 1 of Construction 5.7 the set $\mathcal{D}$ is formed, and it has $\beta\left(C_{p} \times C_{q}\right)-\beta\left(C_{p}\right)-1=$ $p q-1$ diamonds.

Now we move on to Step 2. We computed $\mathcal{C}(\overleftrightarrow{G}) / \mathcal{V}$ in Example 5.1. Recall that $\mathcal{C}(\overleftrightarrow{G}) / \mathcal{V}=\left\{\mathcal{V}, A_{1}+\mathcal{V}, A_{2}+\mathcal{V}, A_{3}+\mathcal{V}\right\}$. where $A_{1}$ and $A_{2}$ are opposite orientations on $C_{p}$, and $A_{3}=A_{1}+A_{2}$ is an anti-cycle of length $2 p$. Note that $w_{q}\left(A_{1}\right)=w_{q}\left(A_{2}\right)=q$, and $w_{q}\left(A_{3}\right)=2 p$. Depending on whether $q<2 p$ or $2 p<q$, we would choose as our minimum $q$-weight basis either $\mathcal{A}=\left\{A_{1}+\mathcal{V}, A_{2}+\mathcal{V}\right\}$ or $\mathcal{A}=\left\{A_{1}+\mathcal{V}, A_{3}+\mathcal{V}\right\}$. In the first case, $\widetilde{\mathcal{A}}=\left\{\widetilde{A}_{1}, \widetilde{A}_{2}\right\}$ consists of two $q$-cycles. In the second case $\widetilde{\mathcal{A}}=\left\{\widetilde{A}_{1}, \widetilde{A}_{3}\right\}$ consists of one $q$-cycle and one $2 p$-cycle. Notice that the resulting MCB $\widetilde{\mathcal{A}} \cup \mathcal{D}$ agrees with Proposition 4.1.

Now we prove that our construction is valid.

Proof. First we confirm that $\mathcal{B}$ is a basis of $\mathcal{C}\left(G \times C_{q}\right)$. Note that $|\mathcal{B}|=|\widetilde{\mathcal{A}} \cup \mathcal{D}|=$ $(\beta(G)+1)+\left(\beta\left(G \times C_{q}\right)-\beta(G)-1\right)=\beta\left(G \times C_{q}\right)$, so we just need to show that $\mathcal{B}$ is independent. Index $\mathcal{D}$ as $\mathcal{D}=\left\{D_{d}: 1 \leq d \leq \beta\left(G \times C_{q}\right)-\beta(G)-1\right\}$ and suppose

$$
\sum_{i \in I} \widetilde{A}_{i}+\sum_{d \in \Delta} D_{d}=0,
$$

where $I \subseteq\{1,2, \ldots, \beta(G)+1\}$ and $\Delta$ is a subset of the set that idexes $\mathcal{D}$. We want to show $I=\Delta=\emptyset$. Taking $\pi^{\prime}$ of both sides of Equation (5.1) produces $\sum_{i \in I} \pi^{\prime}\left(\widetilde{A}_{i}\right)=\mathcal{V}$, which by choice of the $\widetilde{A}_{i}$ becomes $\sum_{i \in I}\left(A_{i}+\mathcal{V}\right)=\mathcal{V}$, so $I=\emptyset$. Thus $\sum_{d \in \Delta} D_{d}=0$ by Equation (5.1), but since $\mathcal{D}$ is linearly independent by construction we have $\Delta=\emptyset$. Thus $\mathcal{B}$ is a basis.

To prove that $\mathcal{B}$ is minimal, consider any $C \in \mathcal{C}\left(G \times C_{q}\right)$ and put

$$
C=\sum_{i \in I} \widetilde{A}_{i}+\sum_{d \in \Delta} D_{d} .
$$


According to Proposition 1.1, we just need to show that $|C|$ is not smaller than the length of any term in this sum. Certainly $|C| \geq\left|D_{d}\right|=4$ for each $d \in \Delta$ because the condition $\max \{p, q\}>3$ implies that $G \times C_{q}$ has no triangles, and hence no cycles of length smaller than 4. We just need to confirm $|C| \geq\left|\widetilde{A}_{i}\right|$ for each $i \in I$. We will do this in cases.

Case 1. Suppose $|C| \geq q$. Take $\pi^{\prime}$ of both sides of (5.2) to obtain

$$
\pi(C)+\mathcal{V}=\sum_{i \in I}\left(A_{i}+\mathcal{V}\right)
$$

Let $S$ be the maximum symmetric sub-digraph of $\pi(C)$. (So $S=0$ if $\pi(C)$ has no double edges.) Then $\pi(C)=Y+S$ where $Y$ consists of all the arcs of $\pi(C)$ that are not arcs of $S$. (So $Y=0$ if $\pi(C)$ is symmetric.) Thus $Y$ has no double edges, so it is an orientation of the eulerian subgraph $f(Y)$ of $G$. (Recall that $f$ is the function that "erases" the orientation of $Y$.) Now, $f(Y)$ decomposes into a disjoint union of simple cycles, so $Y=\sum_{j=1}^{n} B_{j}$ where the $B_{j}$ are orientations on pairwise disjoint simple cycles of $G$. For each index $j$, let $B_{j}^{\prime}$ be an orientation on $f\left(B_{j}\right)$ such that $B_{j}^{\prime}$ is a simple directed odd cycle if $\left|B_{j}\right|$ is odd, or an anti-cycle if $\left|B_{j}\right|$ is even. Then $B_{j}+B_{j}^{\prime}$ is symmetric for each $j$, so $S+\sum_{j=1}^{n}\left(B_{j}+B_{j}^{\prime}\right)$ is symmetric, though it may or may not be in $\mathcal{V}$. Let $B_{0}$ be a shortest odd (simple) cycle in $G$, and let $B_{0}^{\prime}$ be an orientation of $B_{0}$ such that $B_{0}^{\prime}$ is a directed odd cycle. Let $B_{-1}^{\prime}$ be the orientation of $B_{0}$ that is opposite to the orientation of $B_{0}^{\prime}$ (i.e. $B_{-1}^{\prime}$ is $B_{0}^{\prime}$ with the arcs reversed). Thus $B_{0}^{\prime}+B_{-1}^{\prime}$ is a symmetric digraph with $p$ (odd) double edges. Observe

$$
b\left(B_{-1}^{\prime}+B_{0}^{\prime}\right)+S+\sum_{j=1}^{n}\left(B_{j}+B_{j}^{\prime}\right) \in \mathcal{V}
$$

where $b$ is 0 or 1 according to whether the symmetric digraph $S+\sum_{j=1}^{n}\left(B_{j}+B_{j}^{\prime}\right)$ has an even or odd number of double edges. Since $\pi(C)=S+Y=S+\sum_{j=1}^{n} B_{j}$, the above equation tells us

$$
\pi(C)+\mathcal{V}=b\left(B_{-1}^{\prime}+\mathcal{V}\right)+b\left(B_{0}^{\prime}+\mathcal{V}\right)+\sum_{j=1}^{n}\left(B_{j}^{\prime}+\mathcal{V}\right)
$$

Recall that each $B_{j}^{\prime}$ in this sum is either an anti-cycle or a simple directed odd cycle. Since $|C| \geq q \geq p$ we have $|C| \geq q=\max \left\{q,\left|B_{0}^{\prime}\right|\right\}=w_{q}\left(B_{0}^{\prime}\right)$, and similarly $|C| \geq w_{q}\left(B_{-1}^{\prime}\right)$. Also for $1 \leq j \leq n$ we have $|\pi(C)| \geq\left|B_{j}^{\prime}\right|$ by construction, so $|C| \geq \max \{q,|\pi(C)|\} \geq$ $\max \left\{q,\left|B_{j}^{\prime}\right|\right\} \geq w_{q}\left(B_{j}\right)$. Therefore

$$
|C| \geq w_{q}\left(B_{j}^{\prime}\right) \text { for }-1 \leq j \leq n \text {. }
$$

Now for each $-1 \leq j \leq n$ we have $B_{j}^{\prime}+\mathcal{V}=\sum_{i \in J_{j}}\left(A_{i}+\mathcal{V}\right)$ for an appropriate index set $J_{j}$. Also it must be the case that

$$
w_{q}\left(B_{j}^{\prime}\right) \geq w_{q}\left(A_{i}\right) \text { for every } i \in J_{j},
$$

for if $w_{q}\left(B_{j}^{\prime}\right)<w_{q}\left(A_{i}\right)$ for some $i$ and $j$ we could exchange element $A_{i}+\mathcal{V}$ of basis $\mathcal{A}$ with $B_{j}^{\prime}+\mathcal{V}$, contradicting the fact that $\mathcal{A}$ is a minimum $q$-weight basis. Using Equation 
(5.4), we get

$$
\begin{aligned}
\pi(C)+\mathcal{V} & =b\left(B_{-1}^{\prime}+\mathcal{V}\right)+b\left(B_{0}^{\prime}+\mathcal{V}\right)+\sum_{j=0}^{n}\left(B_{j}^{\prime}+\mathcal{V}\right) \\
& =b \sum_{i \in J_{-1}}\left(A_{i}+\mathcal{V}\right)+b \sum_{i \in J_{0}}\left(A_{i}+\mathcal{V}\right)+\sum_{j=0}^{n} \sum_{i \in J_{j}}\left(A_{i}+\mathcal{V}\right)
\end{aligned}
$$

Comparing this with (5.3) and using (5.5) and (5.6), it follows that $|C| \geq w_{q}\left(A_{i}\right)=\left|\widetilde{A}_{i}\right|$ for each $i \in I$.

Case 2. Suppose $|C|<q$. Then $C$ must be a cycle in $G \times\left(C_{q}-e\right)$ for some edge $e \in E\left(C_{q}\right)$. By Lemma 3.3 there is an edge $a b \in E\left(C_{q}-e\right)$ for which

$$
C=A+D
$$

where $A \in \mathcal{C}(G \times a b) \subseteq \mathcal{C}\left(G \times C_{q}\right)$ and $D \in \mathcal{D}\left(G \times\left(C_{q}-e\right)\right) \subseteq \mathcal{D}\left(G \times C_{q}\right)$, and $|C| \geq|A|$.

We claim that $\pi(A)$ is an anti-cycle: WLOG assume $b=a+1$. Now, the degree of any vertex $(x, a)$ of $A$ is even, so let its neighbors be $\left(y_{i}, b\right)$ for $1 \leq i \leq 2 k$ for some integer $k$. Then the set of arcs of form $\pi\left((x, a)\left(y_{i}, b\right)\right)=\overrightarrow{x y_{i}}$ are the outward-pointing arcs at the vertex $x$ of $\pi(A)$, so the out-degree of $x$ is even. Similarly, since the degree of vertex $(x, b)$ of $A$ is even, the same argument shows that the in-degree of $x$ is even. Thus $\pi(A)$ is an anti-cycle. Observe also that $|A|=|\pi(A)|$ because any arc $\overrightarrow{x y}$ of $\pi(A)$ can be the image of only one edge $(x, a)(y, b)$ of $A$. Since $|C| \geq|A|$, we also have $|C| \geq|\pi(A)|=w_{q}(\pi(A))$.

Write $\pi(A)+\mathcal{V}$ as

$$
\pi(A)+\mathcal{V}=\sum_{j \in J}\left(A_{j}+\mathcal{V}\right),
$$

and observe that we must have $w_{q}(\pi(A)) \geq w_{q}\left(A_{j}\right)$ for each $j \in J$, for otherwise some element $A_{j}+\mathcal{V}$ of the basis $\mathcal{A}$ could be exchanged for $\pi(A)+\mathcal{V}$, violating the fact that $\mathcal{A}$ is a minimal $q$-weight basis. Now take $\pi^{\prime}$ of both sides of Equation (5.2) to get $\pi^{\prime}(C)=$ $\sum_{i \in I}\left(A_{i}+\mathcal{V}\right)$. Since $\pi^{\prime}(C)=\pi^{\prime}(A+D)=\pi^{\prime}(A)=\pi(A)+\mathcal{V}$, we have $\pi(A)+\mathcal{V}=$ $\sum_{i \in I}\left(A_{i}+\mathcal{V}\right)$. Comparing this with Equation (5.8), we have $I=J$. Since we have established $|C| \geq w_{q}(\pi(A)) \geq w_{q}\left(A_{j}\right)=\left|\widetilde{A}_{j}\right|$ for all $j \in J$, we now also have $|C| \geq\left|\widetilde{A}_{i}\right|$ for all $i \in I$. This completes the proof.

This concludes our solution to the special case $G \times C_{q}$ of the general problem of constructing an MCB for $G \times H$ in terms of invariants of the factors. We believe the reader may now have a sense of the complexity of such a general construction. We maintain hope that someone will find a simple construction for the general case, though we expect that such a construction would involve elements of our approach.

As noted in the introduction, the MCB problem has been resolved for the Cartesian, strong and lexicographic products. To our knowledge, the modular product (see Appendix $\mathrm{C}$ of [6]) is the only associative product that remains unexplored.

\section{References}

[1] F. Berger, Minimum Cycle Bases of Graphs, dissertation, Technische Universität München, 2004. 
[2] Z. Bradshaw and M. M. M. Jaradat, Minimum cycle bases for direct products of $K_{2}$ with complete graphs, Australasian J. Comb., 43 (2009), 127-131.

[3] R. Diestel, Graph Theory, third ed., Springer-Verlag, Berlin, 2005.

[4] R. Hammack, Minimum cycle bases of direct products of bipartite graphs, Australasian J. Comb. 36 (2006), 213-221.

[5] R. Hammack, Minimum cycle bases of direct products of complete graphs, Information Processing Letters 102 (4) (2007), 214-218.

[6] W. Imrich and S. Klavžar, Product Graphs; Structure and Recognition, Wiley Interscience Series in Discrete Mathematics and Optimization, New York, 2000.

[7] W. Imrich and P. Stadler, Minimum cycle bases of product graphs, Australasian J. Comb. 26 (2002), 233-244.

[8] M. M. M. Jaradat, Minimum cycle bases of lexicographical products of graphs, Discussions Mathematicae Graph Theory 28 (2) (2008), 229-247.

[9] M. M. M. Jaradat, On the basis number of the direct product of graphs, Australasian J. Comb., 27 (2003), 293-306.

[10] D. J. A. Welsh, Kruskal's theorem for matroids, Proc. Cambridge Phil. Soc. 64 (1968), 3-4. 